\title{
Towards a Time-of-Flight Positron Emission Tomography System Based on Multi-Pixel Photon Counter Read-out
}

\author{
Etiennette Auffray, Erika Garutti, Martin Göttlich, Tobias Harion, Pierre Jarron, Paul Lecoq, Thomas Meyer, \\ Francois Powolny, Hans-Christian Schultz-Coulon, Wei Shen, Alessandro Silenzi, Rainer Stamen, \\ Alexander Tadday
}

\begin{abstract}
We present the first commissioning data of our TOFPET test device featuring MPPC read-out of small size LFS crystals. We discuss the characteristics of the test device and present results from two different multi-channel read-out ASIC chips which offer a high timing performance.
\end{abstract}

\section{INTRODUCTION}

$\mathbf{T}$ HE aim of our work is to build and commission a multichannel time-of-flight Positron Emission Tomography (TOF-PET) test device with Silicon Photomultiplier (MultiPixel Photon Counter from HAMAMATSU) read-out featuring a coincidence time resolution of about 300 ps FWHM. This corresponds to an improvement by a factor of two compared to commercially available devices which offer a time resolution of about 600 ps (see for example [1]). A coincidence time resolution of $300 \mathrm{ps}$ allows to locate the origin of the two back-to-back $511 \mathrm{keV}$ Gamma rays with a precision of $4.5 \mathrm{~cm}$ FWHM. It has been shown that TOF information of this precision significantly improves the image quality (see for example [2]). While for two channel systems coincidence time resolutions of 240 ps FWHM are achieved [3], it still has to be proven that similar results can be obtained with a multi-channel system with Silicon Photomultiplier read-out. Our TOF-PET device is meant to serve as a test bench for different multi-channel read-out electronics with high timing performance.

Apart from the challenging requirements on the time resolution we are aiming for highly granular PET detectors in order to obtain a high spatial resolution. Due to their compact design Silicon Photomultipliers coupled to small size scintillating crystals allow the design of detectors with unprecedented granularity.

In the present work we first discuss the design of the TOFPET test device and present the characterization measurements

Manuscript received November 12, 2010

This work is supported by the Helmholtz-Nachwuchsgruppen grant VHNG-206 and the BMBF, grant no. 05HS6VH1.

E. Garutti, M. Göttlich and A. Silenzi are with DESY, Notkestr. 85, D22607 Hamburg, Germany

E. Auffray, P. Jarron, P. Lecoq, T. Meyer and F. Powolny are with CERN, CH-1211 Geneva 23, Switzerland

T. Harion, H.-C. Schultz-Coulon, W. Shen, R. Stamen and A. Tadday are with University of Heidelberg, Im Neuenheimer Feld 227, D-69120 Heidelberg, Germany

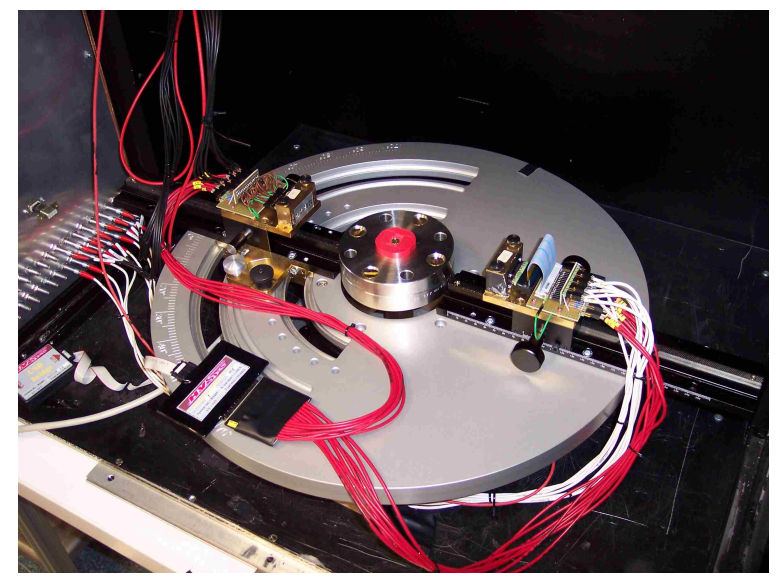

Fig. 1. The TOF-PET test device featuring two PET detectors on a turn table.

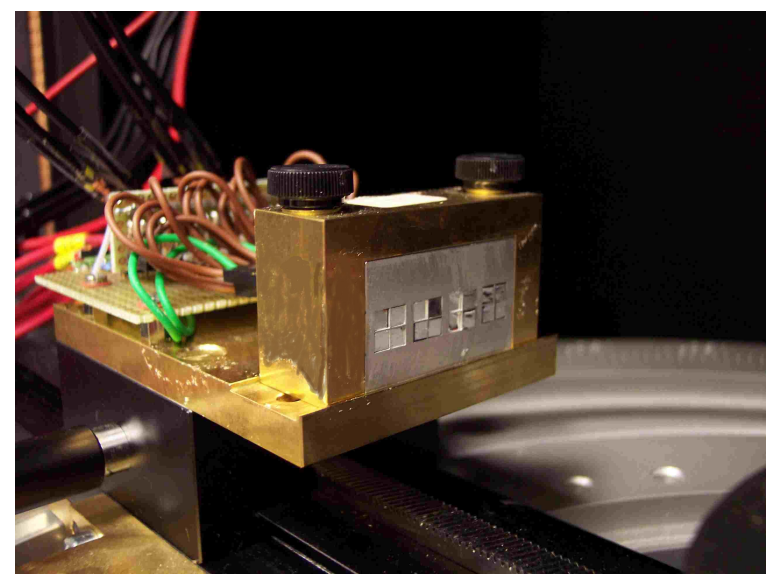

Fig. 2. One of the two PET detector modules of the test device consisting of four submodules. Each submodule consists of a matrix of four crystals read out individually by an MPPC.

we performed. Then we elaborate on two different multichannel read-out alternatives and present the first measurements performed with a two channel system.

\section{DeSIGN OF THE TOF-PET TEST DEVICE}

The prototype of our TOF-PET test device is shown in figure 1. It consists of two modules mounted on a movable support, which allows rotation and translation. Each module 


\begin{tabular}{|l|l|l|l|l|l|}
\hline $\begin{array}{l}\text { Type } \\
\left(\text { size }\left[\mathrm{mm}^{2}\right]\right)\end{array}$ & $\begin{array}{l}\text { Bias } \\
{[\mathrm{V}]}\end{array}$ & $\begin{array}{l}\text { Dark } \\
\text { Rate } \\
{[\mathrm{kHz}]}\end{array}$ & $\begin{array}{l}\text { Pixel } \\
\text { Size } \\
{\left[\mu \mathrm{m}^{2}\right]}\end{array}$ & $\begin{array}{l}\text { Gain } \\
\left(10^{5}\right)\end{array}$ & $\begin{array}{l}\text { PDE } \\
{[\%]}\end{array}$ \\
\hline $\begin{array}{l}\text { single channel } \\
3600 \text { pixels }(3 \times 3)\end{array}$ & 70.4 & 3000 & $50 \times 50$ & 7.5 & $24 \pm 2$ \\
\hline $\begin{array}{l}2 \times 2 \text { array } \\
4 \times 3600 \text { pixels }(3 \times 3)\end{array}$ & 71.0 & 4500 & $50 \times 50$ & 7.5 & $24 \pm 2$ \\
\hline
\end{tabular}

TABLE I

Properties of the MPPC devices (HAMAMATSU) used in the present study. Dark rate, gain and photon detection efficiency (PDE) are given at nominal voltage. The PDE quoted here is the peak value (in the blue spectral region, i.e. $450 \mathrm{~nm}$ ).

consists of four submodules. A submodule is a $2 \times 2$ array of $3 \times 3 \times 15 \mathrm{~mm}^{3}$ Lutetium Fine Silicate (LFS [4]) crystals which is read out by a $2 \times 2$ MPPC array from HAMAMATSU. LFS has similar properties as LSO in terms of the emission spectrum, the light yield, the decay time $(40 \mathrm{~ns})$ and the density. Each MPPC is read out individually, for a total number of 16 channels per module. It is foreseen to build two additional modules to reduce the scan time which will give 64 read-out channels in total. The modules (see figure 2) are made of aluminium. The cavities for the crystals are eroded by electrical discharge machining so that the individual crystals are separated by a $100 \mu \mathrm{m}$ thick wall. The aluminium provides a very good reflectivity, hence, a good energy resolution is achieved.

The bias voltage is provided by a multi-channel high voltage power supply [5] which allows to adjust the bias voltage for each MPPC device individually. The HV module is connected via a USB bridge to the USB port of a standard personal computer.

A 16-channel 10-bit $100 \mathrm{MHz}$ Flash ADC board with signal processing core (ADCM-16 [6]) is used for the data acquisition. It is integrated on a CCB-PCI carrier board and utilizes one PCI slot of a computer. The device is capable to sustain high acquisitions rates up to a few hundred kHz. For the studies presented in this work only one crystal layer per module is read out, i.e. 8 channels for each module. We use this device only for a basic characterization of the system, as due to the $10 \mathrm{~ns}$ sampling the expected time resolution is not sufficient for our purpose. In the present study we use Multi-Pixel Photon Counter from HAMAMATSU [7] to read out scintillating crystals (LFS). The MPPC shows a high sensitivity in the $450 \mathrm{~nm}$ spectral region. This matches the emission spectra of fast crystal scintillators which are peaked in the blue and ultra-violet spectral region, resulting in a high photon yield. The most important properties of the devices are listed in table I. The quoted photon detection efficiency (PDE) is taken from [8]. Single devices and $2 \times 2$ array devices are used. The former are used for studies on the energy and time resolution, the latter for the PET test device.

We studied the energy resolution of an LFS/MPPC system where the crystal is wrapped in Teflon tape and the MPPC is optically coupled to the crystal using optical grease. As $\beta^{+}$ emitter a ${ }^{22} \mathrm{Na}$ source is used. The signal from the MPPCs is digitized without any amplification. The charge integration is performed by a VME QDC CAEN V965A module (gate

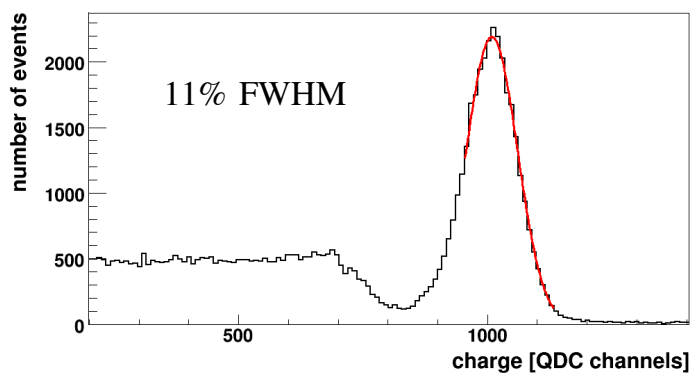

Fig. 3. Energy spectrum of a $511 \mathrm{keV}$ Gamma ray obtained with an LFS crystal (Teflon wrapping) read out by an MPPC.

width $300 \mathrm{~ns})$. Figure 3 shows the measured energy spectrum of $511 \mathrm{keV}$ Gamma rays from a ${ }^{22} \mathrm{Na}$ source. The energy resolution amounts to $11 \%$ FWHM which ranks among the best results reached so far using SiPM devices attached to similar crystals [9], [10], [11], [3], [12], [13]. The mean number of pixels fired is about 2000 for photo-electric events.

We are using the GEANT4 simulation toolkit ([14], [15]) in order to study the theoretical limit on the coincidence timing precision of a two channel system using LFS crystals. Note that we only consider the statistics of the scintillation process but not effects of the photo-detector and the read-out electronics. The best timing precision can be reached when triggering on the first photon. Therefore we consider here the time jitter of the arrival time of the first photon as a measure for the theoretical limit on the timing precision. Since the scintillation rise time plays an important role for the timing properties (cf. [16], [17]) we modified GEANT4 to take the rise time into account. Figure 4 shows the theoretical limit on the coincidence time resolution as a function of the number of photons detected in each of the two photo-detectors for two different scintillation rise times: 500 ps (blue dashed line) and $100 \mathrm{ps}$ (red solid line). Recent measurements indicate that a rise time of $100 \mathrm{ps}$ is realistic [18]. The decay time we used in the simulation is $40 \mathrm{~ns}$. Provided we obtain a high enough photon collection efficiency we see that we are in principle able to obtain a time resolution of 300 ps FWHM with low noise read-out electronics which allows to trigger on the first detected photon. Assuming a realistic time jitter of $160 \mathrm{ps}$ FWHM originating from the combined system of photo-detector and read-out electronics and assuming a total number of pixels fired of 1500 for each of the two detectors we obtain a limit of 260 ps FWHM (black dot in figure 4). Note that for this estimate we assumed a scintiallation rise time of 100 ps.

\section{DETECTOR CHARACTERIZATION}

For the individual channels of the TOF-PET test device we get an average energy resolution of $14 \%$ FWHM with a $6 \%$ channel to channel variation. The slightly inferior result compared to what we get for Telfon wrapped crystals can be mainly attributed to the lower reflectivity of aluminum resulting in a lower photon collection efficiency. The measured timing precision ( $870 \mathrm{ps}$ ) from waveform analysis is limited 


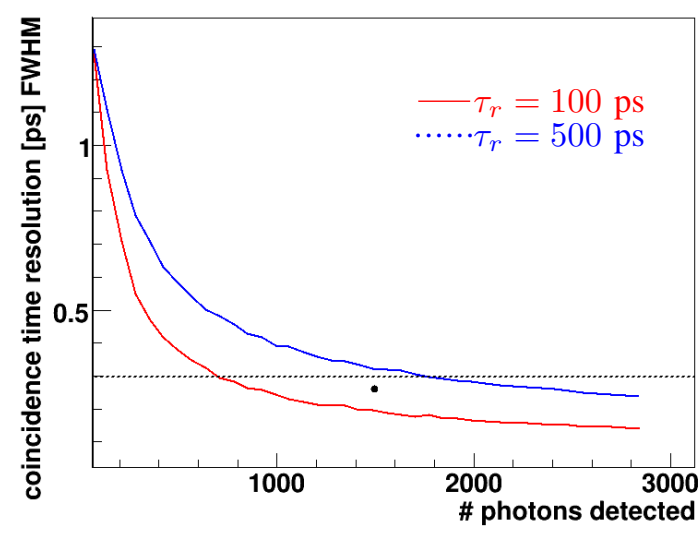

Fig. 4. Theoretical limit on the coincidence time resolution according to GEANT4 as a function of the number of photons detected in each of the two photo-detectors for two different scintillation rise times: 500 ps (blue line) and $100 \mathrm{ps}$ (red line). The black dot is the limit assuming an additional time jitter of 160 ps FWHM originating from the photo-detector and the read-out electronics.

by the relatively low sampling rate (10 ns) of the flash ADC. This is not sufficient for TOF-PET and explains the necessity to develop dedicated multi-channel read-out electronics. This topic will be addressed in the next section.

Figure 6 shows the mean of the photo-electric peak for the individual channels. Applying the same voltage to all MPPC devices results in large differences between the channels (dashed histogram) which can be compensated by voltage adjustments (solid histogram). The errors denote the one sigma region around the mean. Differences between the channels arise mainly due to different breakdown voltages of the MPPC devices and variations in the crystal dimensions and in the coupling to the sensors. The HV cell is equipped with a temperature sensor which allows to automatically adjust the bias voltage of the individual sensors in order to achieve long term stability of the device. Figure 7 shows the temperature dependence of the gain for an MPPC device used in the prototype. By adjusting the bias voltage we are able to stabilize the gain over a wide temperature range (triangles). This is crucial for the timing precision we are aiming for since variations of the gain would cause variations in the effective trigger threshold. Note that we obtain the best timing precision by triggering on the first photon.

In order to study the spatial resolution of the test device we use a radioactive compound consisting of two ${ }^{22} \mathrm{Na}$ sources with a diameter of about $1 \mathrm{~mm}$ and an activity of about $1 \mathrm{MBq}$ each. The distance between the sources is about $10 \mathrm{~mm}$. The compound is placed in the centre of the device and a scan is performed. The following free parameters define a complete scan: 1) The distance of the modules to the centre of the field of view $R$. 2) The number of angular steps $N$ and the size of the steps in degrees $\Delta \phi$.3) The acquisition time at each angle step T. 4) The angle between the modules $\alpha$ which is set to $180^{\circ}$ for the present work. The first two parameters have to be chosen such that large gaps in the angular acceptance are avoided which would deteriorate the image quality. Table II summarizes the results of different scans at different radii and

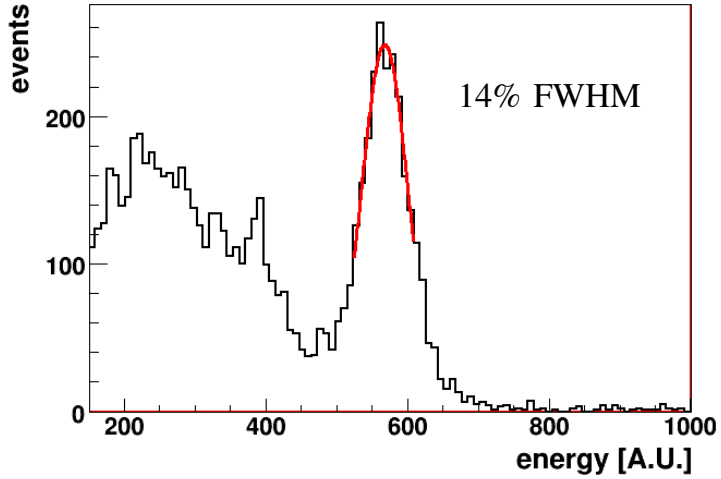

Fig. 5. Energy spectrum obtained with the TOF-PET test device (using the ADCM-16 waveform digitizer).

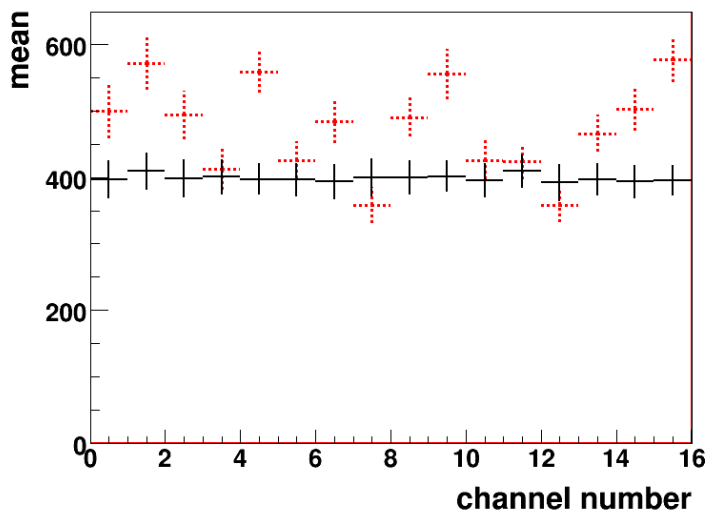

Fig. 6. Mean of the photo-electric peak for the individual channels of one module before (dotted line) and after voltage adjustments (solid line).

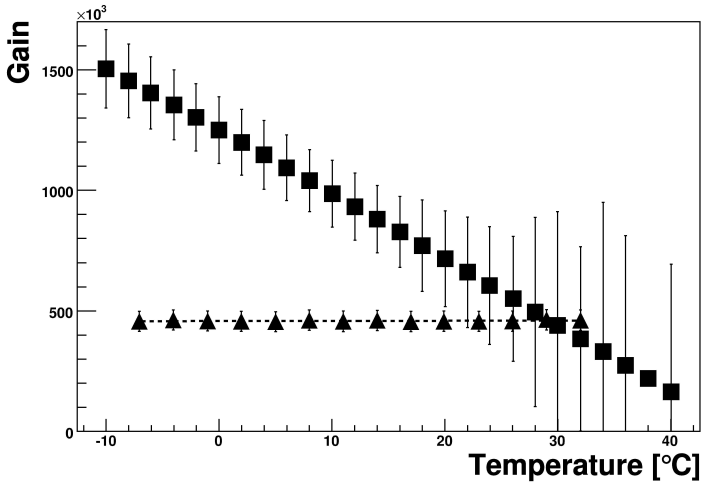

Fig. 7. Temperature dependence of the gain for an MPPC device used in the prototype (squares). By increasing the bias voltage with rising temperature we achieve a stable gain over a wide temperature range (triangles). 


\begin{tabular}{|l|l|l|l|l|}
\hline $\begin{array}{c}R \\
{[\mathrm{~mm}]}\end{array}$ & $N$ & $\begin{array}{l}\Delta \Phi \\
{\left[{ }^{\circ}\right]}\end{array}$ & $\begin{array}{l}\text { T } \\
{[\text { minutes }]}\end{array}$ & $\begin{array}{l}\text { Resolution } \\
\text { FWHM }[\mathrm{mm}]\end{array}$ \\
\hline 90 & 20 & 9 & 10 & 2.5 \\
\hline 90 & 10 & 18 & 10 & 2.5 \\
\hline 180 & 21 & 8.5 & 20 & 2.3 \\
\hline
\end{tabular}

TABLE II

The results in terms of image resolution for different scans and different radii and different steps.

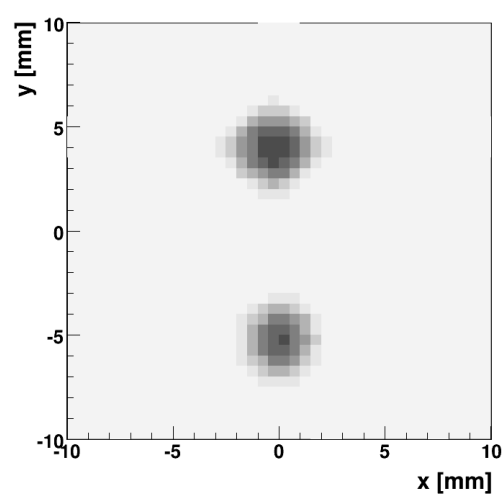

Fig. 8. Reconstructed image of two point sources using the Filtered Backprojection algorithm. The pixel size is $0.5 \times 0.5 \mathrm{~mm}^{2}$.

\section{different steps.}

Images are reconstructed from the sinogram using the Filtered Backprojection algorithm (FBP) applying a ramp filter with a frequency cut-off. The reconstructed image for the first scan in table II is shown in figure 8 . The two sources are well separated. Figure 9 shows the profile of the reconstructed image along the $\mathrm{x}$-axis (at $y=-5.0 \mathrm{~mm}$ ) through one of the two sources. ${ }^{1}$ According to a Gaussian fit the spatial resolution amounts to $2.5 \mathrm{~mm}$ FWHM. Also shown is the MC prediction (hatched histograms) using the GATE toolkit [19]. As expected the agreement between data and simulation is quite good since the spatial resolution is mainly determined by the geometry, i.e. the crystal size. The aim of the simulation studies is to investigate in a next step the efficiency, sensitivity and acceptance for different detectors designs and use this for optimization studies.

\section{Multi-Channel ReAD-OUt Electronics}

In the following we discuss two different multi-channel read-out options which are under development. Both aim for a high timing precision and are able to trigger on the first photon arriving at the detector. Measurements are performed on a two channel system.

\section{A. Measurements with an ultra-fast amplifier-discriminator ASIC (NINO)}

In this section we summarize the results of our measurements with an 8-channel ultra-fast amplifier-discriminator ASIC, the NINO chip. More detailed information about the

\footnotetext{
${ }^{1}$ We define a right-handed Cartesian coordinate system with the $\mathrm{z}$-axis aligned along the rotation axis and the origin in the centre between the modules.
}

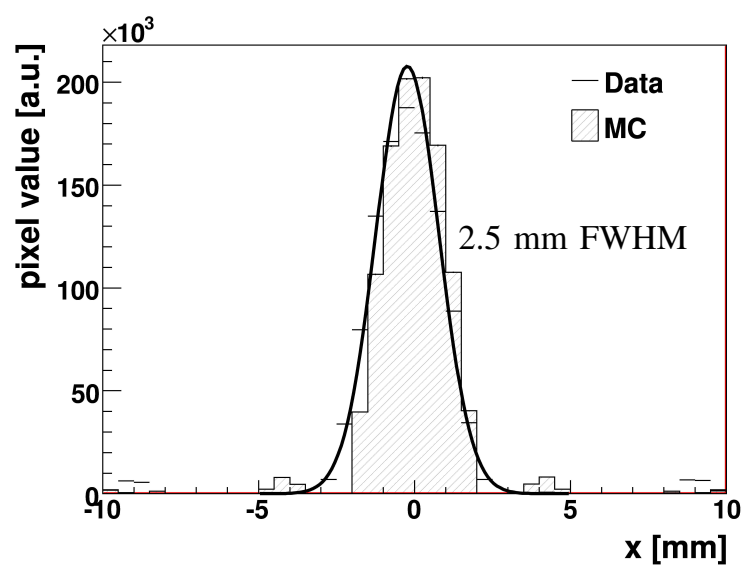

Fig. 9. Profile of the reconstructed image along the x-axis. Also shown is the result of the simulation (hatched histograms). Entries outside the signal region are artifacts from the image reconstruction.

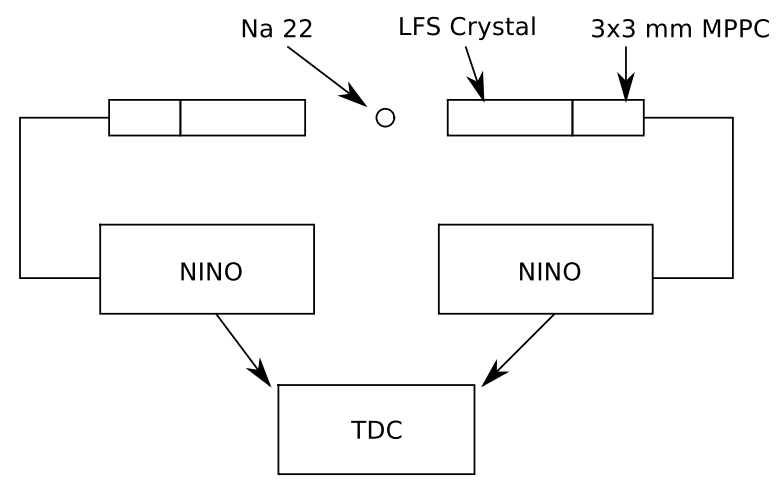

Fig. 10. Experimental setup for the two channel measurement performed with the NINO ASIC.

NINO ASIC and the results presented here can be found in [20] and [21]. The NINO ASIC is used in the TOF system of the ALICE experiment at the LHC. It works as a leading edge discriminator that also encodes, via time over threshold, the energy with the width of the digital output pulse. The anode and the cathode of the MPPC devices are both read out and connected to the differential input of the NINO. Within the NINO the inputs are amplified and parsed by a leading edge discriminator stage employing time over threshold processing. The leading edge of the output signal (Low Voltage Differential Signaling, LVDS) provides the time stamp which can be corrected for time walk via the output pulse width because the time walk depends on the energy.

In order to investigate the coincidence time resolution which can be achieved with the NINO ASIC we used the experimental setup as shown in figure 10. The NINO output is processed by a 16 channel multi-hit VME TDC (CAEN V1290N) with a time resolution of 25 ps (High Performance Time to Digital Converter, HPTDC [22]). The TOT spectra for both channels are shown in figure 11. The energy resolution amounts to about $20 \%$ FWHM which is sufficient to discriminate against Compton events. Selecting only photo-electric events a coincidence time resolution of 350 ps FWHM is reached (see figure 12). The effective trigger threshold corresponds to one fired pixel. In order to put the result of the coincidence time resolution into 


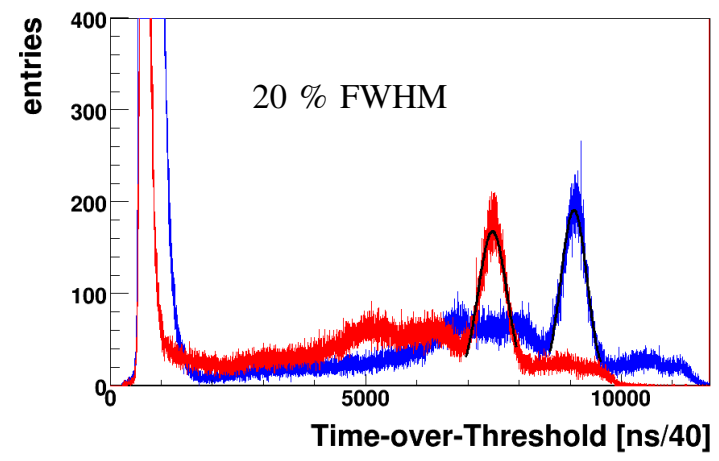

Fig. 11. Time-over-Threshold spectra for the two channels (NINO ASIC).

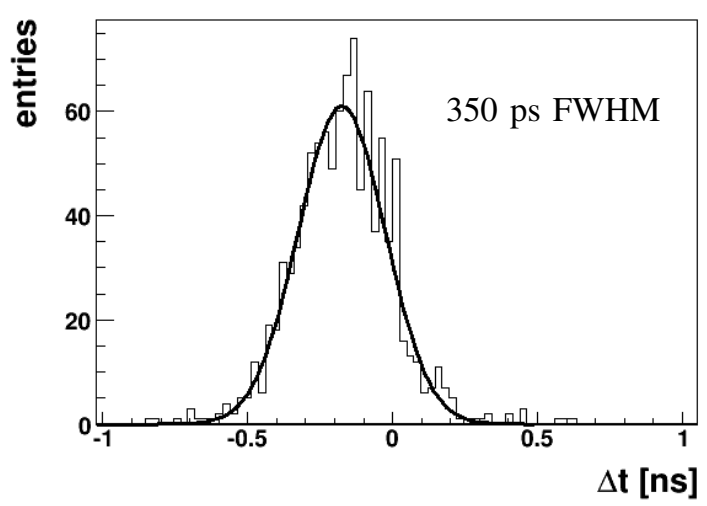

Fig. 12. Delay between the two triggers provided by the NINO ASIC. Shown is a fit to the data, yielding a coincidence time resolution of 350 ps FWHM.

context with measurements performed by other groups and with our simulation results we are still evaluating the photon yield and the time jitter introduced by the photo-detector and the read-out electronics. The data strongly suggests that the limiting factor of our experimental setup is the low light yield.

\section{B. A fast discriminator ASIC dedicated to MPPC read-out (STiC)}

A prototype ASIC (STiC, AMS $0.35 \mu \mathrm{m}$ CMOS technology, cf. [23]), has been designed and its timing precision has been investigated. The prototype chip has 4 channels in total. Each channel of the chip is capable to deliver a trigger signal using two different methods: constant fraction $(\mathrm{CF})$ or leading edge (LE) discrimination. In the present work we only consider the LE method. Due to the very low effective threshold which corresponds to about one pixels fired, the time walk effect inherent to leading edge triggering can be neglected. Note that we only consider high energy (photo-electric) events. Leading edge triggering is accomplished by a low threshold current comparator which has a jitter of about 60 ps. A capacitor in parallel to the input stage of the differentiator can be switched on to measure the charge of the input signal. Each channel has a special DAC to tune the bias voltage at the input signal line, which is used to compensate both, the breakdown voltage variations of the MPPCs and the temperature dependence of the gain. This is an important feature the StiC ASIC offers in

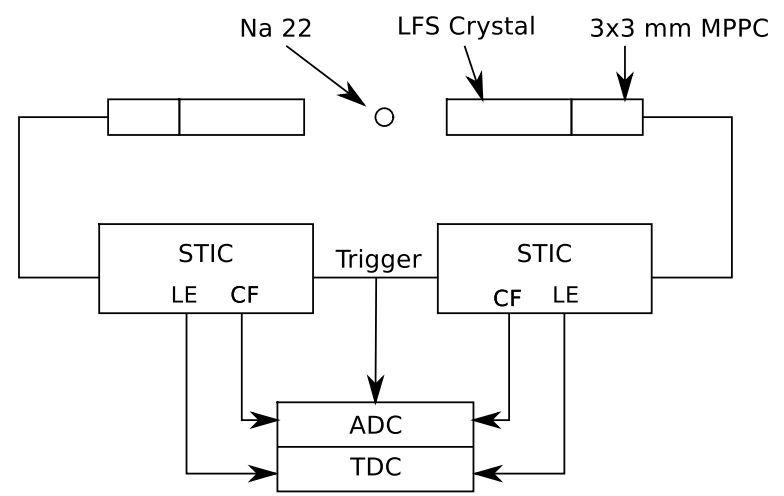

Fig. 13. Experimental setup for the coincidence measurements performed with the STiC ASIC.

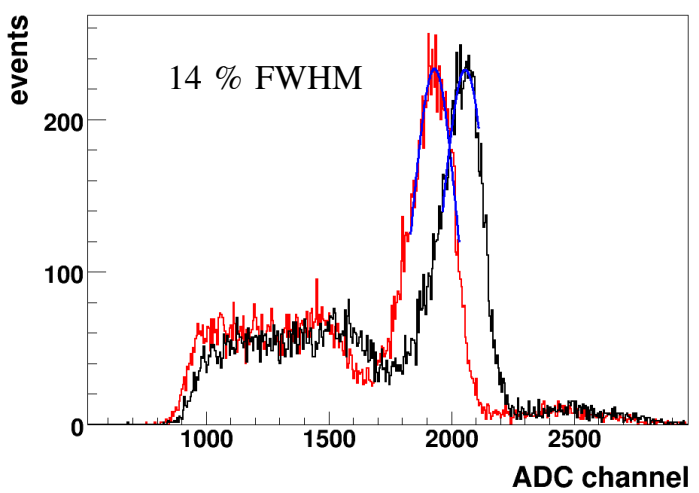

Fig. 14. Energy spectra for the two channels using the STiC ASIC in LE mode.

comparison to the NINO ASIC. All discrimination threshold DACs as well as the bias DACs are controlled by an SPI (Serial Peripheral Interface Bus) slow control interface. The total power consumption is lower than $10 \mathrm{~mW}$ for each individual channel.

We measured the ${ }^{22} \mathrm{Na}$ energy spectrum using the charge integration method. The experimental setup is shown in figure 13. The same two-channel crystal/MPPC system as for the NINO measurement is used. The measured energy spectra are shown in figure 14. An energy resolution of about 14\% FWHM is observed. For the ToT method, a resolution of around $20 \%$ is obtained. The latter result depends very much on the threshold value set for the ToT measurement: The higher the threshold, the better the energy resolution. For the LE method we get a coincidence time resolution of about 480 ps FWHM as depicted in figure 15.

Large electronic noise was observed during the measurements. Several modifications to the design are foreseen for the next STiC version which are expected to considerably improve the timing precision. For details we refer to [24].

\section{CONCLUSIONS AND OUTLOOK}

We have built a compact and uncomplex PET test device featuring MPPC read-out, and presented the first characterization measurements. The prototype consists of arrays of $3 \times 3 \times 15 \mathrm{~mm}^{3}$ LFS crystals read out individually by MPPC 


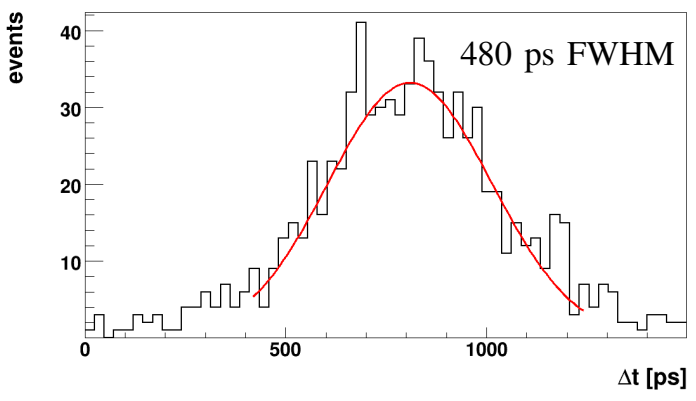

Fig. 15. Delay between the two triggers delivered by the STiC ASIC.

devices with an active area of $3 \times 3 \mathrm{~mm}^{2}$. For a basic characterization of the system the read-out is performed by a 16-channel 10-bit flash ADC board with a sampling rate of $100 \mathrm{MHz}$. A mean energy resolution for $511 \mathrm{keV}$ photons of about $14 \%$ FWHM with a $6 \%$ spread for the various channels is achieved. We were able to reconstruct the position of two point sources close to the centre of the field of view and derived a spatial resolution of $2.5 \mathrm{~mm}$ FWHM. The spatial resolution is mainly determined by the crystal width of $3 \mathrm{~mm}$. Studies of the spatial resolution as a function of the distance to the centre of the field of view are foreseen. We achieved an initial coincidence time resolution of $870 \mathrm{ps}$ FWHM, which is limited by the sampling rate of the ADC. We demonstrated that with a relatively simple approach a very good performance in terms of spatial resolution can be achieved. In addition, we showed that we can reach a very good channel-to-channel homogeneity and stability of the system by monitoring the temperature and adjusting the MPPC bias voltages individually.

In order to improve the coincidence time resolution to better than 300 ps FWHM we have to develop dedicated multi-channel read-out electronics which we will test on our prototype device. Two read-out alternatives are investigated. With an ultra-fast amplifier/discriminator ASIC (NINO) a coincidence time resolution of $350 \mathrm{ps}$ FWHM is reached. The prototype of the STiC ASIC was also tested. Problems leading to a worse time resolution compared to the NINO chip were identified and triggered changes in the design.

\section{ACKNOWLEDGMENT}

We thank Igor Tiapkin for introducing us to the use of the ADCM-16 module and Petr Smirnov (DESY) for his help in the realization of the readout electronics.

\section{REFERENCES}

[1] S. Surti, A. Kuhn, M. E. Werner, A. E. Perkins, J. Kolthammer, J. S Karp, "Performance of Philips Gemini TF PET/CT scanner with special consideration for its time-of-flight imaging capabilities." J Nucl Med, vol. 48, pp. 471-480, March 2007.

[2] J. S. Karp, S. Suleman Surti, M. E. Daube-Witherspoon, G. Muehllehner, "Benefit of Time-of-Flight in PET: Experimental and Clinical Results," J Nucl Med, vol. 49, no. 3, pp. 462-470, March 2008.

[3] C.L. Kim, G.C. Wang, "Multi-Pixel Photon Counters for TOF-PET Detector and its challenges," in Nuclear Science Symposium Conference Record, 2008.

[4] Zecotek, LFS Scintillation Material - Product Information. [Online]. Available: www.zecotek.com
[5] HVSys, HV cell for Silicon Detectors - Data sheet. [Online]. Available: www.hvsys.dubna.ru

[6] AFI Electronics, ADCM-16 - Technical manual. [Online]. Available: afi.jinr.ru

[7] Hamamatsu Photonics, MPPC devices - Data sheet. [Online]. Available: www.hamamatsu.com

[8] A. Tadday et al., "Characterisation Studies of Silicon Photomultipliers," Nuclear Instruments and Method in Physics Research A.

[9] A. N. Otte et al., "A test of silicon photomultipliers as readout for PET," Nucl. Instrum. Meth., vol. A545, pp. 705-715, 2005.

[10] G. Llosa et al., "Silicon Photomultipliers and SiPM matrices as Photodetectors in Nuclear Medicine," in Nuclear Science Symposium Conference Record, 2007.

[11] V. C. Spanoudaki et al., "Use of single photon counting detector arrays in combined PET/MR: Characterization of LYSO-SiPM detector modules and comparison with a LSO-APD detector," JINST, vol. 2, p. P12002, 2007.

[12] N. D'Ascenzo, E. Garutti, M. Goettlich, H. C. Schultz-Coulon, and A. Tadday, "Study of Micro Pixel Photon Counter for the Application to Positron Emission Tomography," 2008.

[13] D. R. Schaart, H. T. van Dam, S. Seifert, R. Vinke, P. Dendooven, H. Lohner, and F. J. Beekman, "A novel, SiPM-array-based, monolithic scintillator detector for PET," Physics in Medicine and Biology, vol. 54, no. 11 , pp. 3501-3512, 2009.

[14] S. Agostinelli et al., "GEANT4: A simulation toolkit," Nucl. Instrum. Meth., vol. A506, pp. 250-303, 2003.

[15] J. Allison et al., "Geant4 developments and applications," IEEE Trans. Nucl. Sci., vol. 53, p. 270, 2006.

[16] M. Kelbert, I. Sazonov, and A. G. Wright, "Exact expression for the variance of the photon emission process in scintillation counters," Nucl. Instrum. Meth., vol. A564, pp. 185-189, 2006.

[17] Y. Shao, "A new timing model for calculating the intrinsic timing resolution of a scintillator detector," Phys. Med. Biol., vol. 52, pp. 11031117, 2007.

[18] S. Seifert et al., "Accurate Measurements of the Rise and Decay Times of Fast Scintillators with Solid State Photon Counters," in Nuclear Science Symposium Conference Record, 2010.

[19] S. Jan et al., "GATE : A simulation toolkit for PET and SPECT," Phys. Med. Biol., vol. 49, pp. 4543-4561, 2004.

[20] F. Anghinolfi, P. Jarron, F. Krummenacher, E. Usenko, and M. C. S. Williams, "NINO: An ultrafast low-power front-end amplifier discriminator for the time-of-flight detector in the ALICE experiment," IEEE Trans. Nucl. Sci., vol. 51, pp. 1974-1978, 2004.

[21] P. Jarron et al., "Time based readout of a silicon photomultiplier (SiPM) for Time Of Flight Positron Emission Tomography (TOF-PET)," in IEEE-NSS 2009 conference proceedings, 2009.

[22] M. Mota1 et al., "A flexible multi-channel high-resolution Time-toDigital Converter ASIC," in IEEE-NSS 2000 conference proceedings.

[23] W. Shen and H. Schultz-Coulon, "STIC - A Current Mode Constant Fraction Discriminator for Positron Emission Tomography using SiPMs (MPPC)," in IEEE-NSS 2009 conference proceedings, 2009.

[24] W. Shen et al., "STiC - ASIC for Silicon-Photomultiplier Fast Timing Discrimination," in IEEE-NSS 2010 conference proceedings, 2010. 